\title{
Model-Free Sliding Mode Control Method
}

\author{
Agamemnon Crassidis, Raul Mittmann Reis \\ Rochester Institute of Technology \\ 76 Lomb Memorial Drive, Rochester, New York 14623, U.S. \\ alceme@rit.edu; rmr2448@rit.edu
}

\begin{abstract}
In this paper, a model-free sliding mode controller is developed and demonstrated on a second order nonlinear system. The proposed controller is based solely on state measurements and previous control inputs, thus, a system model is not required. The underlying knowledge required about the system is its order and the bounds of the input matrix, if it is non-unitary. In order to handle system uncertainties, a discontinuous term is added to the controller form and is designed using Lyapunov's stability theorem to guarantee asymptotically stability during the reaching phase of the state trajectories. The discontinuous term creates an undesirable effect i.e., chattering, which is impractical for implementation of the controller in actual system types. A time-varying smoothing boundary layer is used to eliminate chattering and is also developed in this work. Finally, the controller is tested using a nonlinear second order system with and without including sensor measurement noise effects simulating real-world effects.
\end{abstract}

Keywords: Model-Free, Sliding Mode Controller, Measurement Noise, Lyapunov

\section{Introduction}

Sliding mode control is a popular robust control scheme for nonlinear systems since it is very effective in handling system uncertainties and disturbances. This technique is based on applying a discontinuous control input which drives the system's states onto a desired sliding surface in the state phase domain. However, an undesirable effect is produced with the nominal form of the controller, i.e., control effort chattering, which can be compensated by applying a smooth boundary layer into the controller form while still achieving tracking stability in the Lyapunov sense.

Due its popularity, many sliding mode control schemes have been previously developed. Laghrouche et al. [3] proposed a higher-order sliding mode control law based on optimal linear quadratic control to handle minimum-phase nonlinear models. Seung-Hi et al. [5] presented a discrete-time sliding mode control using fast output sampling which made it possible to arbitrarily assign the system closed loop eigenvalues. Hay et al. [4] submitted a method called adaptive seeking sliding mode control. This method applies a floating gain to relieve the chattering problem, and the unknown uncertainties and disturbances were adaptively estimated and compensated by the updated sliding control method. Cunha et al. [2] proposed an output-feedback model reference sliding mode control to solve the output tracking problem. The strategy of this method is based on an output-feedback unit vector controller to generate the sliding mode. Lastly, Crassidis and Mizov [1] developed a model-free sliding mode controller, which only relies on state and control input measurements.

The work developed in this paper is similar to the one developed in [1]. The system approach is considerably different though, which results in the development of an alternative and new type of model-free sliding mode control scheme. The proposed sliding mode controller also relies only on state and control input measurements. If there is an input matrix, the bounds of it are required to be known to derive the control law. The controller can be directly applied to linear and nonlinear SISO systems. The outline of this paper is as follows. Section 2 describes the system and the sliding surface. In Section 3, the control law and the switching gain are derived. Section 4 contains two illustrative examples implementing this controller, one without the effect of measurement noise and the other one with measurement noise.

\section{System Description}

Consider a $n^{\text {th }}$ - order single-input-single-output autonomous system, where $n$ is the order of the system. The following equality holds true for the system:

$$
x^{n}=x^{n}+b u-b u_{k-1}-b u+b u_{k-1}
$$


where $x$ is the state vector, $u$ is the control input, $u_{k-1}$ is the previous control input and $b$ is the input matrix. The error parameter between the input and the previous input is defined as:

$$
\varepsilon(u)=-u+u_{k-1}
$$

Substituting the Eq. (2) described above into Eq. (1), the following is obtained:

$$
x^{n}=x^{n}+b u-b u_{k-1}+b \varepsilon(u)
$$

In order to compute the control law, and to avoid an algebraic loop within the controller algorithm, an estimation of the control input error is necessary. Thus, the estimation of the control input error is defined as:

$$
\hat{\varepsilon}(u)=u_{k-1}-u_{k-2}
$$

where $u_{k-2}$ is the previous control input of the previous input. Although the control input error is not known exactly, the error is assumed to be bounded as follows:

$$
\left(1-\sigma_{l}\right) \hat{\varepsilon}(u) \leq \varepsilon(u) \leq\left(1+\sigma_{u}\right) \hat{\varepsilon}(u)
$$

where $\sigma_{u}$ is the upper bound and $\sigma_{l}$ is the lower bound of the estimation error. If the sampling time is sufficiently high, the values of the error bounds will be near zero since the estimation error will be approximately equal to the actual error.

\subsection{Sliding Surface}

A sliding surface for a $n^{\text {th }}$ order single-input-single-output system can be defined as:

$$
s=(d / d t+\lambda)^{n-1} \tilde{x}(t)
$$

where $\tilde{x}$ is the difference between the desired state and the actual state, i.e., $\tilde{x}(t)=x(t)-x_{d}(t)$, and $\lambda$ is the slope of the sliding surface, which is assumed to a positive constant. A second order system will be used as illustrative example in this paper. The following sliding surface is obtained from Eq. (6) for a second order system:

$$
s=\dot{\tilde{x}}+\lambda \tilde{x}
$$

\section{Controller}

There is a set of assumptions that must be satisfied in order to develop the model-free control law. The system must be completely observable and controllable, i.e., all system's states can be measured and are controllable Also, if an nonunity gain is observed for the input, such as shown in Eq. (1), the bounds of gain are assumed to be known. If the assumptions are satisfied, a model-free sliding mode controller can be derived. The controller relies solely on state measurements and previous control inputs to apply a control signal onto the system in order to drive the system's states to the desired states, i.e., obtain perfect tracking. The discontinuous term, used to handle the system's uncertainties, is designed using Lyapunov's direct method to ensure closed loop asymptotically stability in the presence of uncertainties.

\subsection{Control Law}

The control law of the sliding mode controller can be obtained by differentiating Eq. (6) with respect to time and setting the equation equal to zero. This is required to ensure that once the system's states are on the sliding surface, they will remain there. Differentiating Eq. (6) with respect to time and setting the equation equal to zero results in:

$$
\dot{s}=\ddot{\tilde{x}}+\lambda \dot{\tilde{x}}=\left(\ddot{x}-\ddot{x}_{d}\right)+\lambda\left(\dot{x}-\dot{x}_{d}\right)=0
$$


Substituting Eq. (1) into Eq. (8) and writing in terms of the control input, the following control law is obtained:

$$
u=b^{-1}\left[-\lambda\left(\dot{x}-\dot{x}_{d}\right)-\left(\ddot{x}-\ddot{x}_{d}\right)\right]+u_{k-1}-\varepsilon(u)
$$

In order to the controller achieve robustness against system's uncertainties, a discontinuous term is added to Eq. (9):

$$
u=b^{-1}\left[-\lambda\left(\dot{x}-\dot{x}_{d}\right)-\left(\ddot{x}-\ddot{x}_{d}\right)-\eta \operatorname{sgn}(s)\right]+u_{k-1}-\varepsilon(u)
$$

where $\eta$ is a small positive constant and $\operatorname{sgn}(s)$ is the signum function of the sliding surface.

\subsection{Proof of the Control Form:}

To ensure that the system's trajectories will be asymptotically stable during the reaching phase, Lyapunov's direct method is used. Lyapunov's direct method is based on the energy of the system to analyse its stability. Briefly saying, if a positive energy system has a decaying energy rate, the system will be stable in Lyapunov sense. Thus, a function that describes the system's energy must be determined, which for this case is defined as:

$$
V(x)=(0.5) s^{2}
$$

which is clearly positive definite, which means that the system have positive energy. To obtain the energy rate of the system, Eq. (11) can be simply differentiated with respect to time:

$$
\dot{V}(x)=\dot{s} s \leq 0
$$

Substituting Eq. (1), Eq. (7) and Eq. (10) into Eq. (12), the following equation is obtained:

$$
\dot{V}(x)=-\eta|s| \leq 0
$$

Since $\eta$ can only assume positive values, the negative-definiteness of Eq. (13) is guaranteed, therefore the closed loop system will be asymptotically stable.

\subsection{Switching Gain}

The control law, described at Eq. (10), is now redefined as:

$$
\hat{u}=\hat{b}^{-1}\left[-\lambda\left(\dot{x}-\dot{x}_{d}\right)-\left(\ddot{x}-\ddot{x}_{d}\right)-K \operatorname{sgn}(s)\right]+u_{k-1}-\hat{\varepsilon}(u)
$$

where $K$ is the switching gain and $\hat{b}$ is the input gain estimation, which is computed by $\hat{b}=\sqrt{b_{u p} b_{\text {low }}}$. In order to derive a switching gain that ensures closed-loop stability during the reach phase, the sliding condition described at Eq. (13) must be satisfied. Therefore, substituting Eq. (12) into Eq. (13) yields:

$$
s s \leq-\eta|s|
$$

Using the definition of the sliding surface, system model, and the control law, the following switching gain is obtained for a second order system:

$$
K=|\beta-1|\left|\ddot{x}-\ddot{x}_{d}\right|+|\beta-1| \lambda\left|\dot{x}-\dot{x}_{d}\right|+\mid \hat{b}\left(\sigma_{u}\left(u_{k-2}-u_{k-1}\right) \mid+\beta \eta\right.
$$

where $=\sqrt{b_{u p} / b_{\text {low }}}$. Also, the control law can be written as follows, using the definition of the estimation error Eq. (4):

$$
\hat{u}=\hat{b}^{-1}\left[-\lambda\left(\dot{x}-\dot{x}_{d}\right)-\left(\ddot{x}-\ddot{x}_{d}\right)-K \operatorname{sgn}(s)\right]+2 u_{k-1}-u_{k-2}
$$


Note that if the input gain parameter is unitary, the control law and the switching gain is simplified to:

$$
\begin{gathered}
K=\left(\sigma_{u}\left(u_{k-2}-u_{k-1}\right)+\eta\right. \\
\hat{u}=\left[-\lambda\left(\dot{x}-\dot{x}_{d}\right)-\left(\ddot{x}-\ddot{x}_{d}\right)-\operatorname{Ksgn}(s)\right]+2 u_{k-1}-u_{k-2}
\end{gathered}
$$

\subsection{Boundary Layer}

In order to reduce the chattering effect, introduced by the discontinuous term, a smoothing layer is included in the control law. The smoothing control discontinuity essentially assigns a low pass filter to the dynamics of the sliding surface, which eliminates the chattering. In order to maintain attractiveness of the boundary layer, the sliding condition, Eq. (13) is updated to as follows:

$$
|s| \geq \phi \rightarrow 0.5\left(d s^{2} / d t\right) \leq(\dot{\phi}-\eta)|s|
$$

where $\phi$ is the boundary layer. Furthermore, a new switching gain must be used in order to satisfy the equation described above as shown by:

$$
\bar{K}=K-\phi
$$

Therefore, the control law becomes:

$$
\hat{u}=\hat{b}^{-1}\left[-\lambda\left(\dot{x}-\dot{x}_{d}\right)-\left(\ddot{x}-\ddot{x}_{d}\right)-\bar{K} s a t(s / \phi)\right]+2 u_{k-1}-u_{k-2}
$$

Where the $\operatorname{sat}(s / \phi)$ function is defined as:

$$
\begin{cases}\operatorname{sat}(s / \phi)=s / \phi & , \text { if }|s / \phi| \leq 1 \\ \operatorname{sat}(s / \phi)=\operatorname{sgn}(s / \phi) & \text {, otherwise }\end{cases}
$$

The dynamics of the boundary layer are determined by:

$$
\dot{\phi}+\lambda \phi=K
$$

where $\phi(0)=\eta / \lambda$. This last equation is also known as the balance condition. With this approach, instead of perfect tracking it ensures a tracking within a known precision.

\section{Simulation}

To validate the sliding mode controller proposed in this paper, a second order system is used as an illustrative example. The plant is a simple mass-spring-damper system. For the first set of simulations the measurement noise is ignored, while for the second one, it is included.

\subsection{Nonlinear without noise example:}

Suppose the following second order nonlinear mass-spring-damper model is to be controlled:

$$
m \ddot{x}+c \dot{x}+k x^{2}=b u
$$

where $m$ is the mass of the system, $c$ is the damping coefficient, $k$ is the spring constant, $u$ is the control input, $b$ is the input matrix, $\ddot{x}, \dot{x}$ and $x$ are the state measurement variables. For this example, the mass is set to $2 \mathrm{~kg}$, the damping coefficient to $0.8 \mathrm{~N} / \mathrm{m} / \mathrm{s}$, the spring constant to $2 \mathrm{~N} / \mathrm{m}$ and the input matrix varies within 1 and 5 . The controller parameters, $\sigma_{u}$ and $\eta$, were defined as $20,0.5$ and 0.1 respectively. 
Using the control law, sliding surface, and the switching gain defined previously, a control system using Simulink and MATLAB was developed. A fixed step size of 0.0001 seconds was used for the 30 second simulation. The tracking problem is to track the reference signal defined as $x_{d}(t)=\sin (\pi t / 2)$. The following results were obtained.
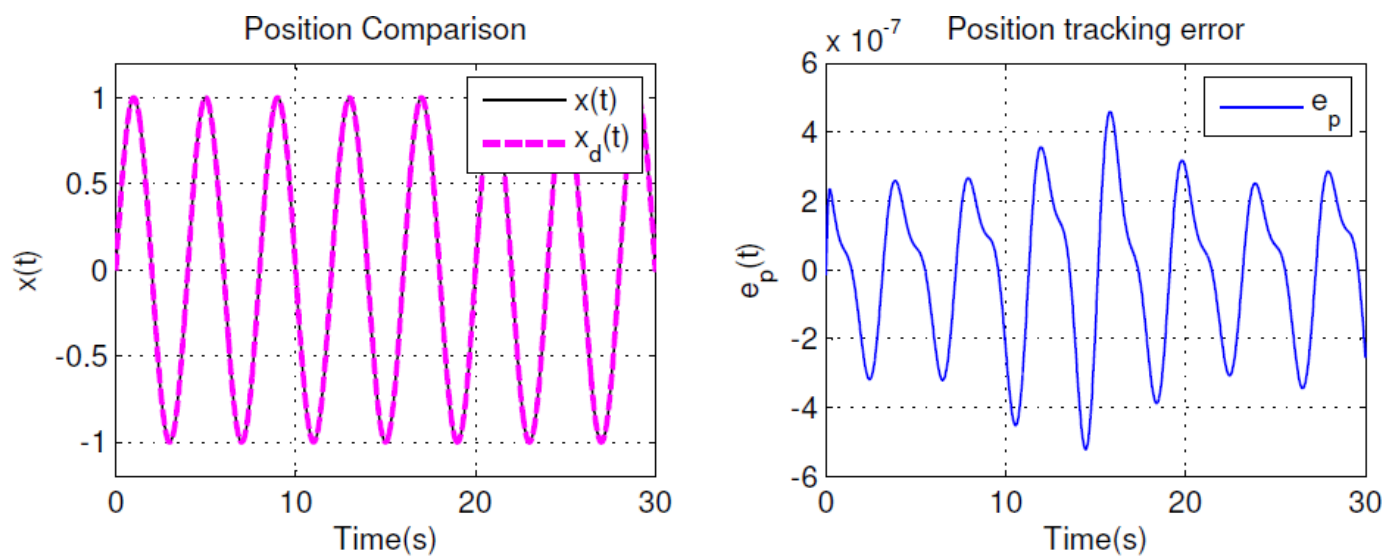

Fig. 1: Position comparison and position tracking error.

Figure 1 displays the position comparison and the position tracking error. The position error is less than 5e-7, which is minimal representing outstanding agreement between the state position measurement and tracking position reference signal. Thus, near perfect tracking is achieved.
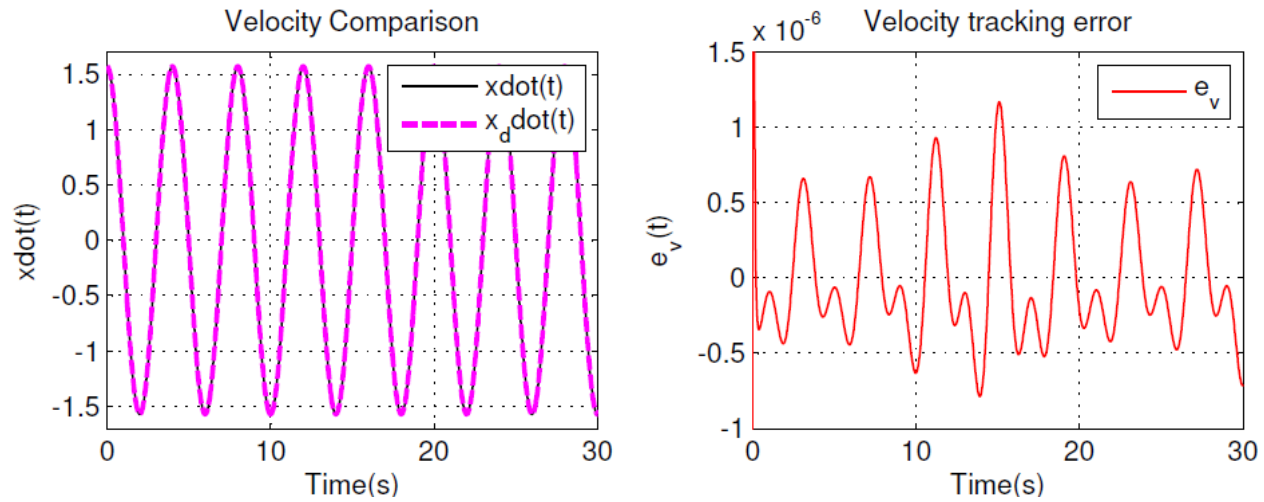

Fig. 2: Velocity comparison and velocity tracking error.

Figure 2 displays the velocity comparison and the velocity tracking error. Once again, near perfect tracking is achieved.
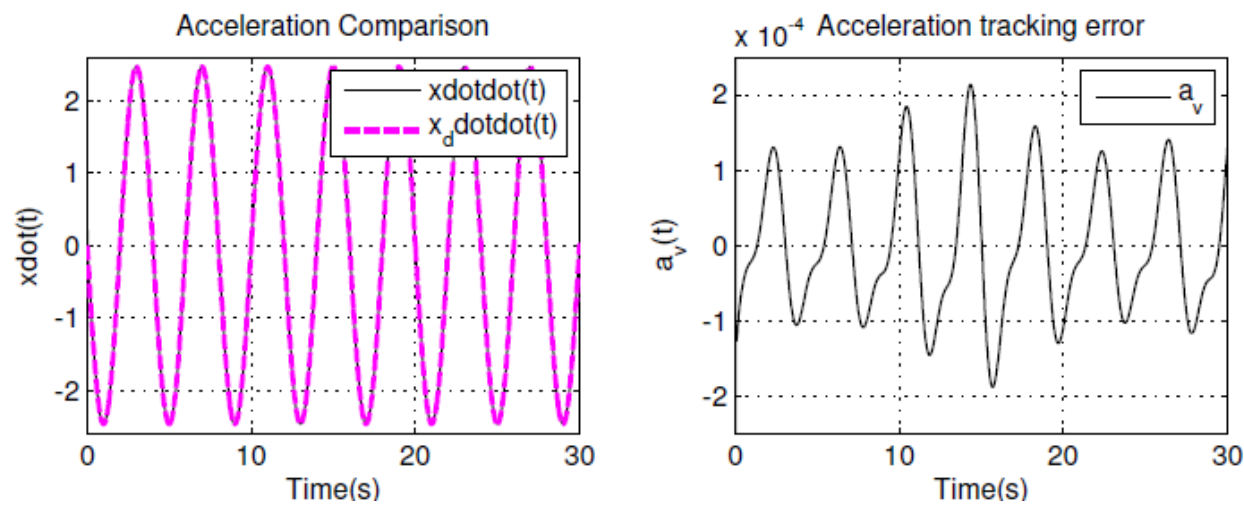

Fig. 3: Acceleration comparison and acceleration tracking error. 
The acceleration comparison and the acceleration tracking error are shown in Figure 3. The error is considerable small, with values less than $3 \mathrm{e}-4$.
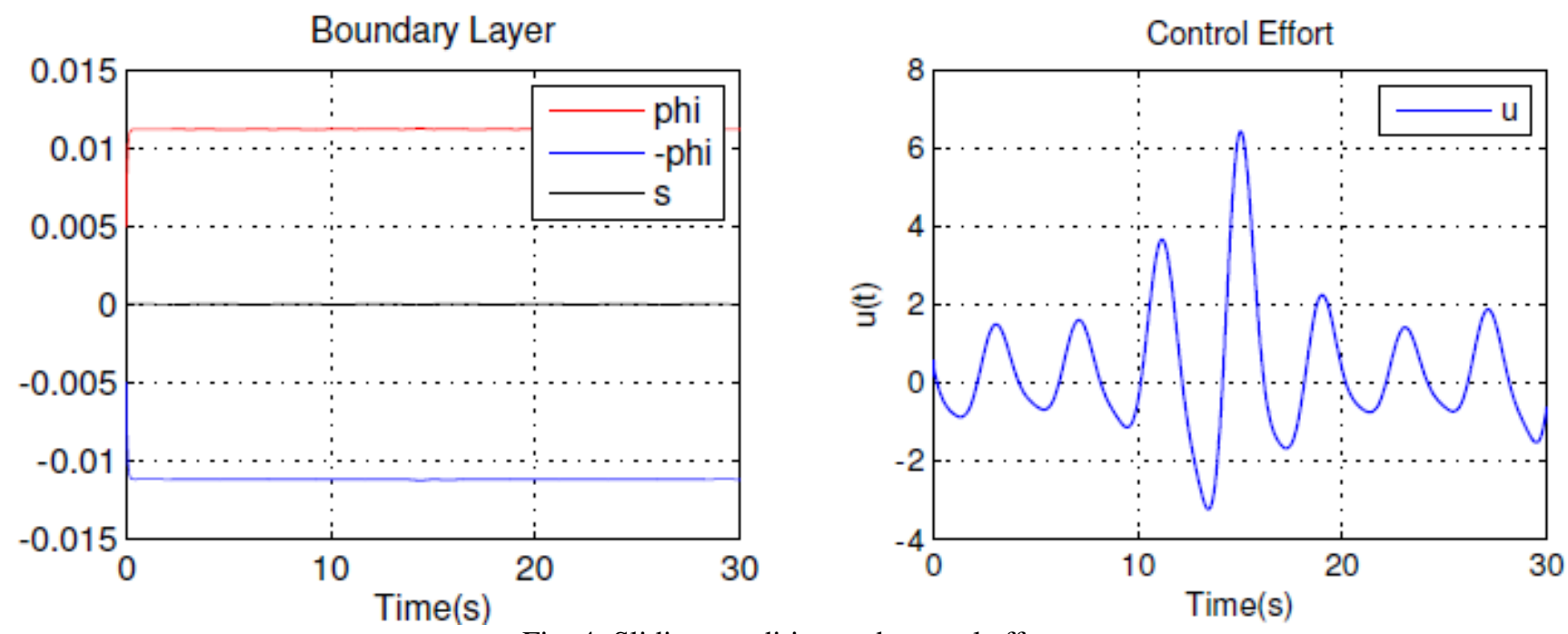

Fig. 4: Sliding condition and control effort.

Lastly, the sliding condition and the control effort are shown in Figure 4. The sliding condition is always satisfied, since the sliding surface remains within the boundary layer. The effect of chattering is eliminated, since the control response is smooth.

\subsection{Nonlinear with noise example:}

The same nonlinear second order system defined at Eq. (25) was used to control the nonlinear system with measurement noise. A Gaussian noise with known variance and zero mean was included to the system's state measurements. The variance, the mean and the probability distribution function of the noise can be obtained using sensor manufacturer's datasheet. Hence, the induced measurement noise can be considered realistic considering commonly used sensors. The $\lambda$ and $\eta$ values of the controller must be modified though. As it can be seen by Eq. (16) and Eq. (17), the $\lambda$ value actuate as a gain for the noise. Hence, if it is too high, the system response will become noisy. Also, it is not feasibility to reduce the size of the boundary layer to ensure a tracking precision smaller than the peak to peak noise value. The following equation is proposed to select $\lambda$ regarding the presence of measurement noise:

$$
\lambda \leq \hat{b}\left(\eta_{0} / V_{p p}\right)^{\frac{1}{n}}
$$

where $\eta_{0}$ is the $\eta$ value used for the system without noise and $V_{p p}$ is the peak to peak noise value. The $V_{p p}$ value can be set in different ways. If the user defines $V_{p p}=3 \sigma_{n}$, where $\sigma_{n}$ is the noise standard deviation, it is guaranteed that $99 \%$ of the time the noise value will be within $V_{p p}$. The $\eta$ value has also to be updated:

$$
\eta=\eta_{0}+\left(\sigma_{n} / \eta_{0}\right)(\lambda / 2)
$$

The same controller used in the previous simulation was used with $\lambda$ and $n_{0}$ set to 1 and 0.1 , respectively. The same reference function was used to track as well. The following results were obtained: 

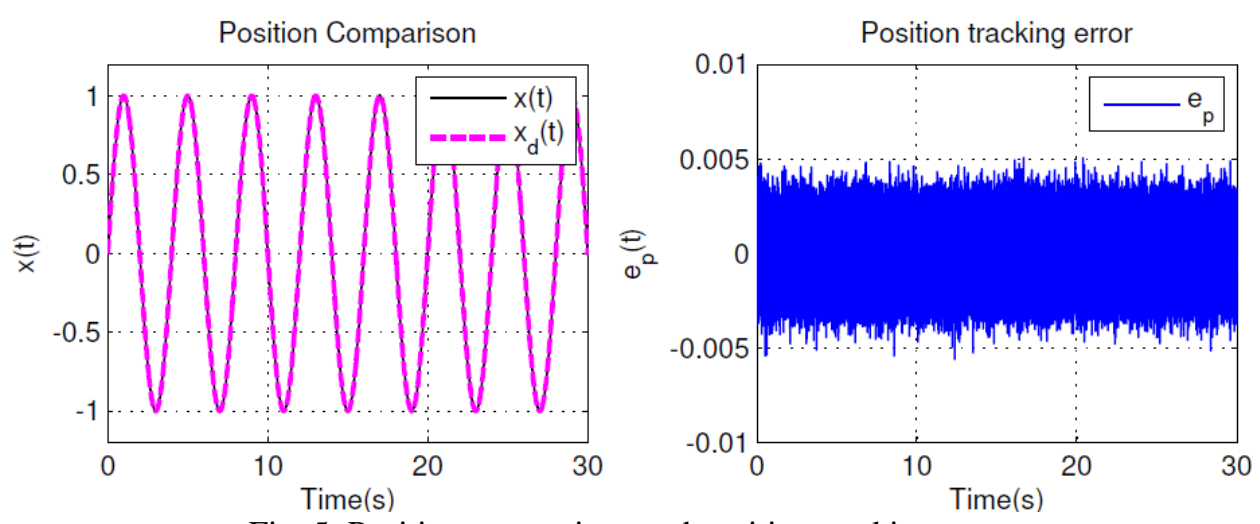

Fig. 5: Position comparison and position tracking error.

Figure 1 displays the position comparison and the position tracking error. The position error is less than $0.01 \mathrm{~m}$, which is nearly equal to the noise measurement representing outstanding tracking response.
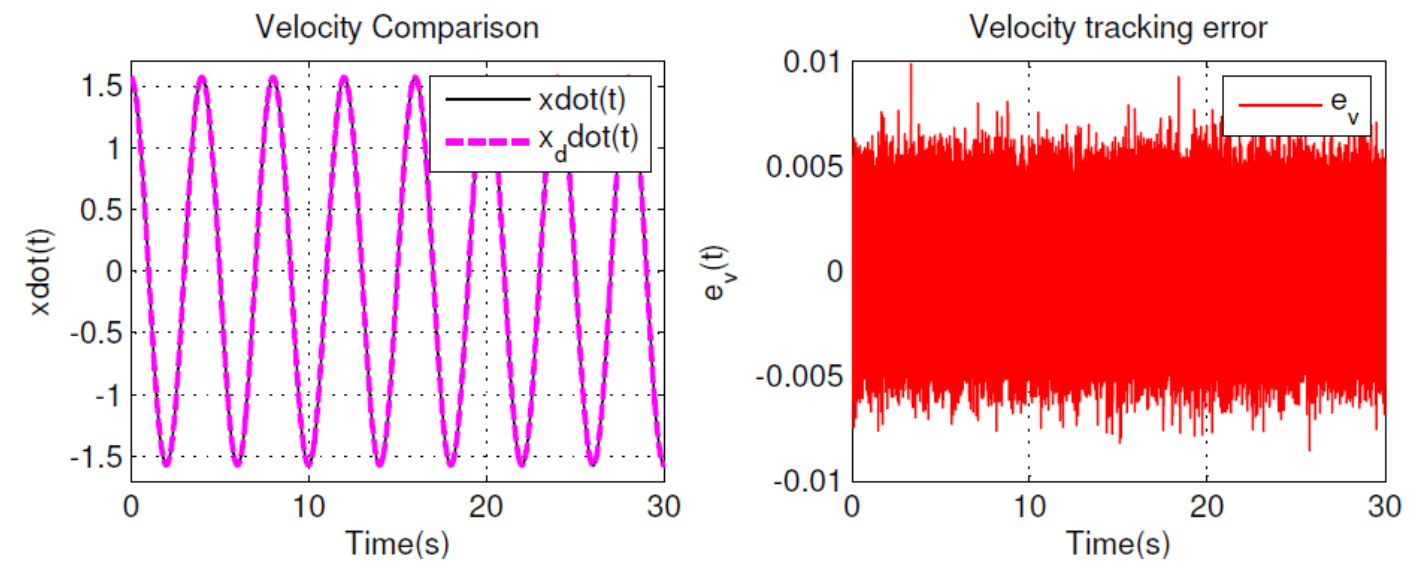

Fig. 6: Velocity comparison and velocity tracking error.

Figure 2 displays the velocity comparison and the velocity tracking error. The velocity tracking error is also due to the noise measurement, with values less than $0.01 \mathrm{~m} / \mathrm{s}$.
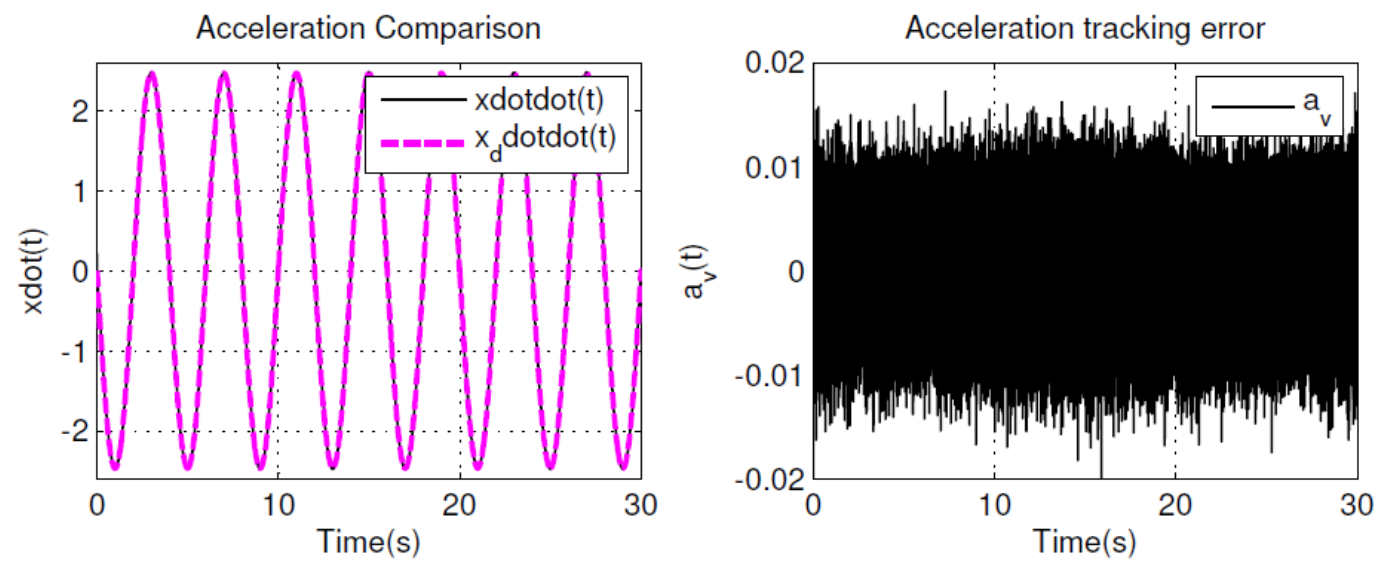

Fig. 7: Acceleration comparison and acceleration tracking error.

Figure 3 displays the acceleration comparison and the acceleration tracking error. The error is less than $0.02 \mathrm{~m} / \mathrm{s} / \mathrm{s}$, but the closed-loop system is achieving an outstanding tracking response in the presence of measurement noise. 

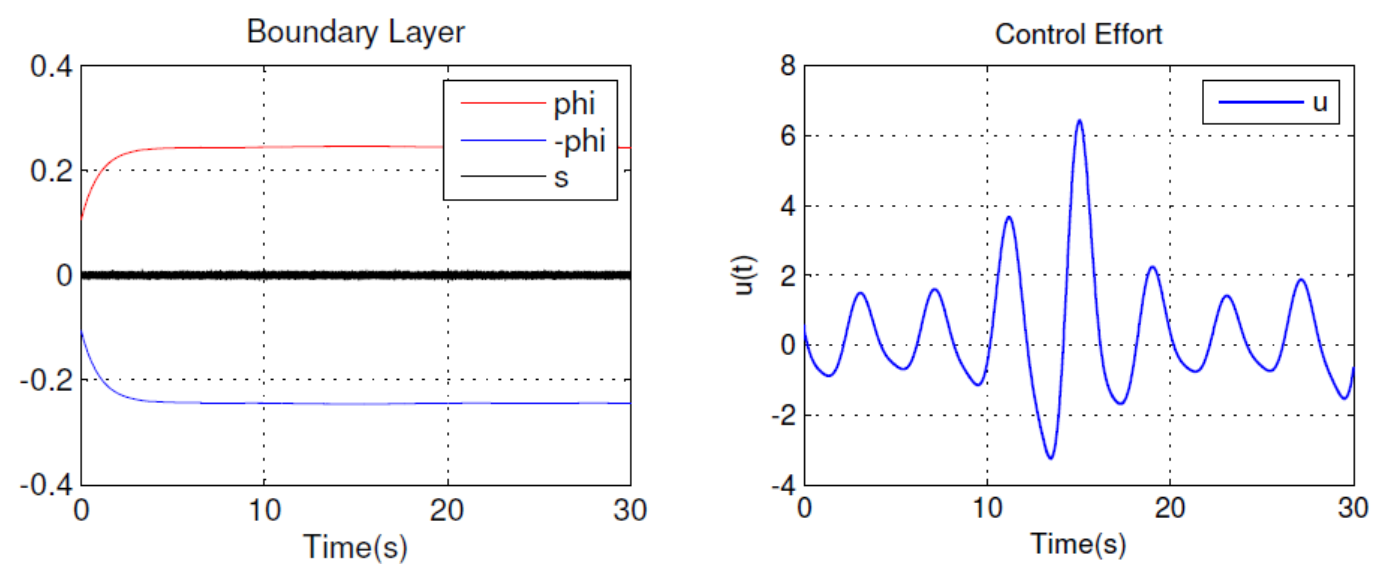

Fig. 8: Sliding condition and control effort.

The sliding condition and the control effort are displayed at Figure 8. The boundary layer is expanded, compared to the previous simulation, so the tracking precision is reduced. However, the sliding surface remains within the boundary layer and the control effort is still smooth.

\section{Conclusion}

A model-free sliding mode control based only in state measurements and previous control inputs was proposed. To reduce the well-known chattering effect, introduced by the discontinuous term of the sliding mode controller, a smoothing boundary layer was inserted in the controller form. The controller was implemented to a second order nonlinear system and was proved to be robust and stable in presence of parameter variation and noise measurement. The control effort response was shown to be smooth and the sliding condition was satisfied all time even in the presence of measurement noise.

\section{References}

[1] Crassidis A. and Mizov A., "A Model-Free Control Algorithm Derived Using the Sliding Mode Control Method," in Proceedings of the $2^{\text {nd }}$ International Conference of Control, Dynamic Systems, and Robotics, Ottawa, ON, 2015, Paper No. 166.

[2] Cunha J.P.V.S, Hsu L., Costa R.R. and Lizarralde F., "Output-Feedback Model-Reference Sliding Mode Control of Uncertain Multivariable Systems," in IEEE Transactions on Automatic Control, 2003, vol. 48, pp. 2245-2250.

[3] Laghrouche S., Plestan F., and Glumineau A., "Higher Order Sliding Mode Control Based on Optimal Linear Quadratic Control," in European Control Conference, Cambridge, UK, 2003, pp. 910-915.

[4] Hay Y. and Ozguner U., "Adaptive Seeking Sliding Mode Control," in American Control Conference, Minneapolis, MN, 2006, pp. 4694-4699.

[5] Seung-Hi L. and Chung C.C, "Sliding Mode Control Design Using Fast Output Sampling," in $42^{\text {nd }}$ IEEE Conference on Decision and Control, Maul, HI, 2003, vol. 4, pp. 3543-3548. 